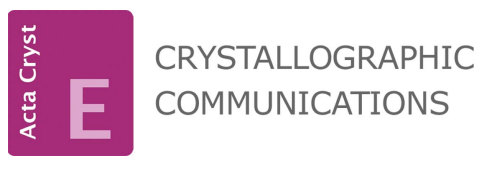

ISSN 2056-9890
Received 26 November 2017

Accepted 20 January 2018

Edited by V. Khrustalev, Russian Academy of Sciences, Russia

Keywords: iron(III) complex; 5-bromo-salicylaldehyde-2-pyridylhydrazone; 5-bromo-salicylaldehyde-thiosemicarbazone; high-spin; magnetic property; crystal structure.

CCDC reference: 1818280

Supporting information: this article has supporting information at journals.iucr.org/e

\section{A new mononuclear neutral high-spin iron(III) complex with the different tridentate ligands 5-bromosalicylaldehyde (pyridin-2-yl)hydrazone and 5-bromosalicylaldehyde thiosemicarbazone}

\author{
Yun Zhao, Xiao-Feng Shen and Li-Fang Zhang*
}

School of Chemical Engineering and Technology, China University of Mining and Technology, Xuzhou 221116, People's Republic of China. *Correspondence e-mail: zhanglifang@cumt.edu.cn

The title neutral mononuclear complex, [1-(5-bromo-2-oxidobenzylidene)thiosemicarbazidato](4-bromo-2-\{[2-(pyridin-2-yl)hydrazinylidene]methyl\}phenolato)iron(III), $\quad\left[\mathrm{Fe}\left(\mathrm{C}_{8} \mathrm{H}_{6} \mathrm{BrN}_{3} \mathrm{OS}\right)\left(\mathrm{C}_{12} \mathrm{H}_{9} \mathrm{BrN}_{3} \mathrm{O}\right)\right]$ (I), crystallizes in the monoclinic space group $\mathrm{C}_{2} / c$ and has two different planar tridentate ligands. The central $\mathrm{Fe}^{\mathrm{III}}$ ion is coordinated to three $\mathrm{N}$, two $\mathrm{O}$ and one $\mathrm{S}$ atom, forming a distorted octahedral $\mathrm{FeN}_{3} \mathrm{O}_{2} \mathrm{~S}$ coordination geometry. In the crystal, the complex molecules are linked by $\mathrm{N}-\mathrm{H} \cdots \mathrm{O}$ and $\mathrm{N}-\mathrm{H} \cdots \mathrm{N}$ hydrogen bonds and $\pi-\pi$ interactions into layers parallel to (100). Magnetic measurements show that the central $\mathrm{Fe}^{\mathrm{III}}$ ion is in the high-spin state; this is also supported by the bond distances around the $\mathrm{Fe}^{\mathrm{III}}$ ion.

\section{Chemical context}

Much attention have been paid to the design and synthesis of $\mathrm{Fe}^{\mathrm{III}}$ complexes for magnetic materials owing to their interesting thermal- or light-induced spin conversion between the high-spin (HS, $S=5 / 2$ ) and low-spin (LS, $S=1 / 2$ ) states (Li et al., 2013; Phonsri et al., 2017; Sato et al., 2007). It is well known that the organic ligands usually play a significant role in the crystal structures and magnetic properties of metal complexes (Ni et al., 2017; Zhang et al., 2016). Up to date, many Fe ${ }^{\mathrm{III}}$ complexes with spin-crossover (SCO) behavior have been designed and synthesized through the subtle design and combination of different ligands. Among the many organic ligands, Schiff bases are the most common ligands for new $\mathrm{Fe}^{\mathrm{III}}$ complexes due to their convenient synthesis and regulation. Compared with homo-ligand complexes, the employment of mixed ligands provides more selection and modification strategies for new magnetic complexes. In previous reports, the ligands 5-bromo-salicylaldehyde-2pyridylhydrazone (5-Br-Hpsal), 5-bromo-salicylaldehydethiosemicarbazone (5-Br- $\mathrm{H}_{2}$ thsa) and their derivatives have been explored to assembly $\mathrm{Fe}^{\mathrm{III}}$ and $\mathrm{Mn}^{\mathrm{III}}$ complexes with SCO behavior (Shongwe et al., 2014). Recently, we obtained the title complex, $\left[\left(\mathrm{C}_{20} \mathrm{H}_{15} \mathrm{~N}_{6} \mathrm{O}_{2} \mathrm{SBr}_{2}\right) \mathrm{Fe}\right](\mathrm{I})$, using 5-Br-Hpsal and 5- $\mathrm{Br}-\mathrm{H}_{2}$ thsa ligands. Herein, we report the crystal structure and magnetic property of this iron(III) complex.

\section{Structural commentary}

The title complex (Fig. 1) crystallizes in the monoclinic space group $C_{2} / c$. Compound (I) is a neutral mononuclear complex 
with two different rigid tridentate ligands $-5-\mathrm{Br}^{-\mathrm{psal}^{-}}$and 5$\mathrm{Br}^{-}$thsa $^{2-}$ - which adopt a meridional coordination mode. The central $\mathrm{Fe}^{\mathrm{III}}$ ion lies almost within the plane of each ligand [give deviations] and is coordinated to three nitrogen, two oxygen and one sulfur atoms from the two tridentate $5-\mathrm{Br}$ $\mathrm{psal}^{-}$and 5-Br-thsa ${ }^{2-}$ ligands, forming a distored octahedral $\mathrm{FeN}_{3} \mathrm{O}_{2} \mathrm{~S}$ geometry. The $\mathrm{Fe}-\mathrm{O}$ bond lengths are 1.943 (3) and 1.931 (3) $\AA$, the $\mathrm{Fe}-\mathrm{N}$ bond lengths range from 2.142 (3) to 2.157 (3) $\AA$, and the Fe1-S1 bond length is 2.4093 (14) $\AA$. All the bond lengths are normal and agree well with those in related high-spin state $\mathrm{Fe}^{\mathrm{III}}$ compounds ( $\mathrm{Li}$ et al., 2013; Phonsri et al., 2017). The $\mathrm{C} 1-\mathrm{S} 1$ bond length $[1.720$ (4) $\AA]$ is comparable with the ordinary $\mathrm{C}-\mathrm{S}$ bond length $(\mathrm{Li} \&$ Sato, 2017), whereas the $\mathrm{C} 1=\mathrm{N} 2$ and $\mathrm{C} 2=\mathrm{N} 3$ bond distances [1.314 (5) and 1.287 (5) $\AA$, respectively] are significantly smaller than those of $\mathrm{C} 1-\mathrm{N} 1[1.350(5) \AA]$ and $\mathrm{C} 16-\mathrm{N} 5$ [1.377 (6) $\AA$ ] indicating the double-bond character. The bond angles further evidence the significantly distorted octahedral coordination geometry around the $\mathrm{Fe}^{\mathrm{III}}$ ion.

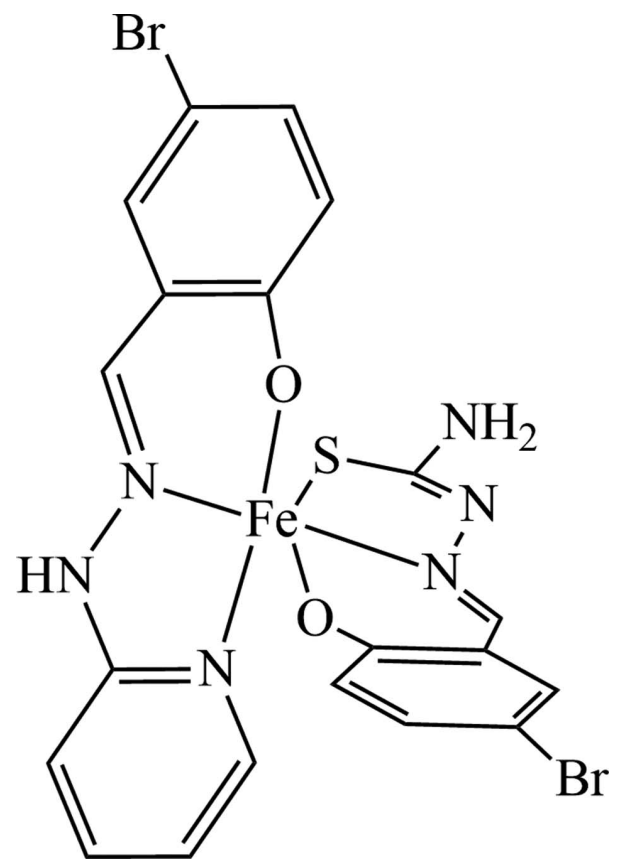

\section{Supramolecular features}

In the crystal, there are two independent hydrogen bonds (Table 1), which link the complex molecules into layers parallel to (100) (Fig. 2). In addition, there exist relatively strong $\pi-\pi$ interactions between the pyridine and benzene rings of the 5 -Br-psal ${ }^{-}$ligands with a shortest interatomic distance of 3.485 (3) $\AA$ (Fig. 2).

\section{Magnetic properties}

The magnetic susceptibilities of (I) have been measured in the temperature range $2-350 \mathrm{~K}$ under an applied magnetic field strength of 2000 Oe by SQUID magnetometry. A plot of $\chi_{\mathrm{m}} T$ versus $T$ is presented in Fig. 3, where $\chi_{\mathrm{m}}$ represents the molar
Table 1

Hydrogen-bond geometry $\left(\AA{ }^{\circ}\right)$.

\begin{tabular}{lllll}
\hline$D-\mathrm{H} \cdots A$ & $D-\mathrm{H}$ & $\mathrm{H} \cdots A$ & $D \cdots A$ & $D-\mathrm{H} \cdots A$ \\
\hline $\mathrm{N} 5-\mathrm{H} 5 \cdots \mathrm{N} 2^{\mathrm{i}}$ & $0.83(4)$ & $2.00(4)$ & $2.825(5)$ & $171(4)$ \\
$\mathrm{N} 1-\mathrm{H} 1 A \cdots \mathrm{O} 2^{\mathrm{ii}}$ & 0.88 & 2.29 & $2.987(4)$ & 136 \\
\hline
\end{tabular}

Symmetry codes: (i) $-x+\frac{1}{2}, y-\frac{1}{2},-z+\frac{1}{2}$; (ii) $x,-y+2, z+\frac{1}{2}$.

magnetic susceptibility per $\mathrm{Fe}^{\mathrm{III}}$ unit. The $\chi_{\mathrm{m}} T$ value is 4.042 emu $\mathrm{K} \mathrm{mol}^{-1}$ at room temperature, which is slightly smaller than the expected value of $4.375 \mathrm{emu} \mathrm{K} \mathrm{mol}^{-1}$ for the single spin carrier of high-spin $\mathrm{Fe}^{\mathrm{III}}(S=5 / 2)$ based on $g=2.0$. The measurement of the magnetic property shows that the $\mathrm{Fe}^{\mathrm{III}}$ ion is in the high-spin state, which agrees well with the abovementioned bond lengths around the $\mathrm{Fe}^{\mathrm{III}}$ ion. The $\chi_{\mathrm{m}} T$ value keeps nearly constant with decreasing temperature until around $75 \mathrm{~K}$, and then it decreases quickly to a minimum value of $1.12 \mathrm{emu} \mathrm{K} \mathrm{mol}^{-1}$ at $2.0 \mathrm{~K}$. This tendency to change of the $\chi_{\mathrm{m}} T$ curve indicates the existence of overall weak antiferromagnetic interactions in (I). The magnetic susceptibilities in the range of 2-350 K comply well with the Curie-Weiss law with a negative Weiss constant $\theta=-4.28 \mathrm{~K}$, and Curie constant $C=4.08 \mathrm{emu} \mathrm{K} \mathrm{mol}{ }^{-1}$, which further confirms the presence of overall intermolecular antiferromagnetic interactions between neighboring $\mathrm{Fe}^{\mathrm{III}}$ ions through intermolecular hydrogen bonds and $\pi-\pi$ interactions in complex (I).

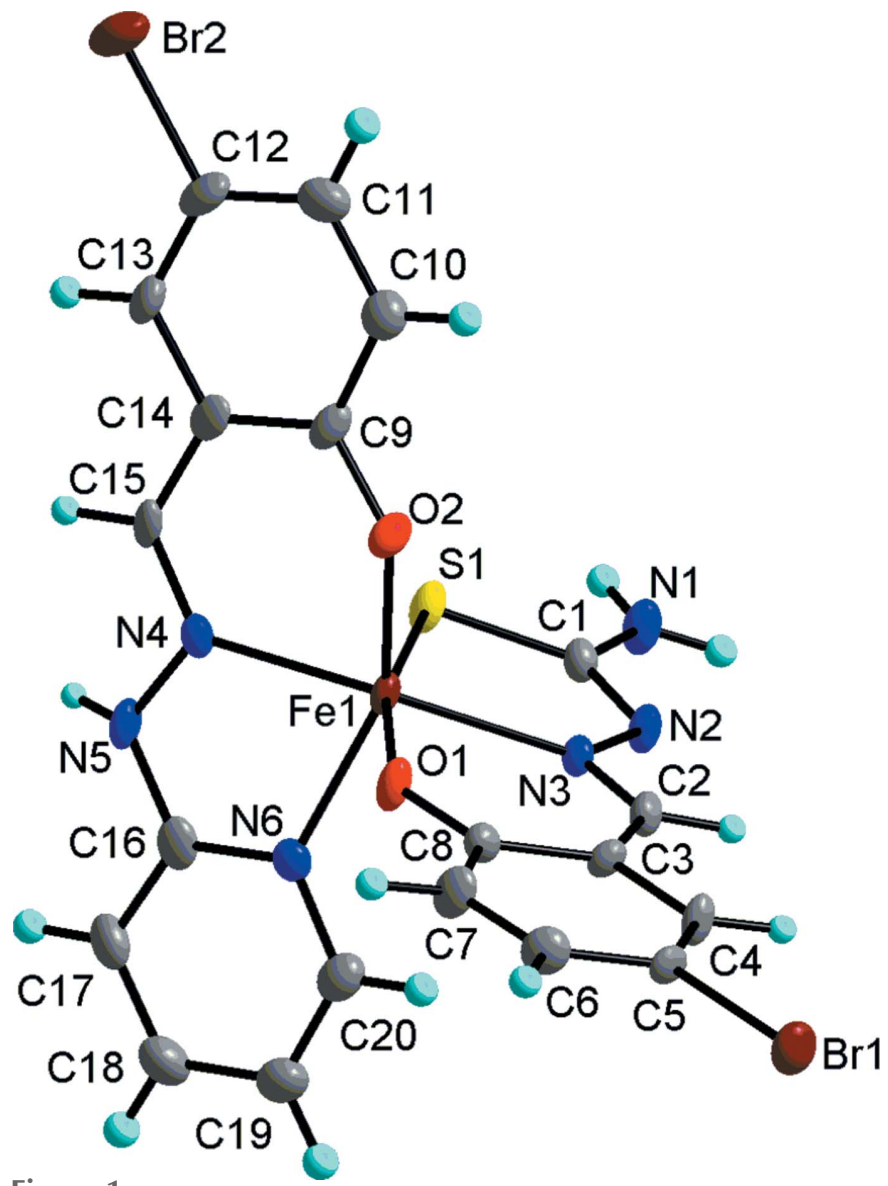

Figure 1

Molecular structure of (I) with $30 \%$ probability displacement ellipsoids. 


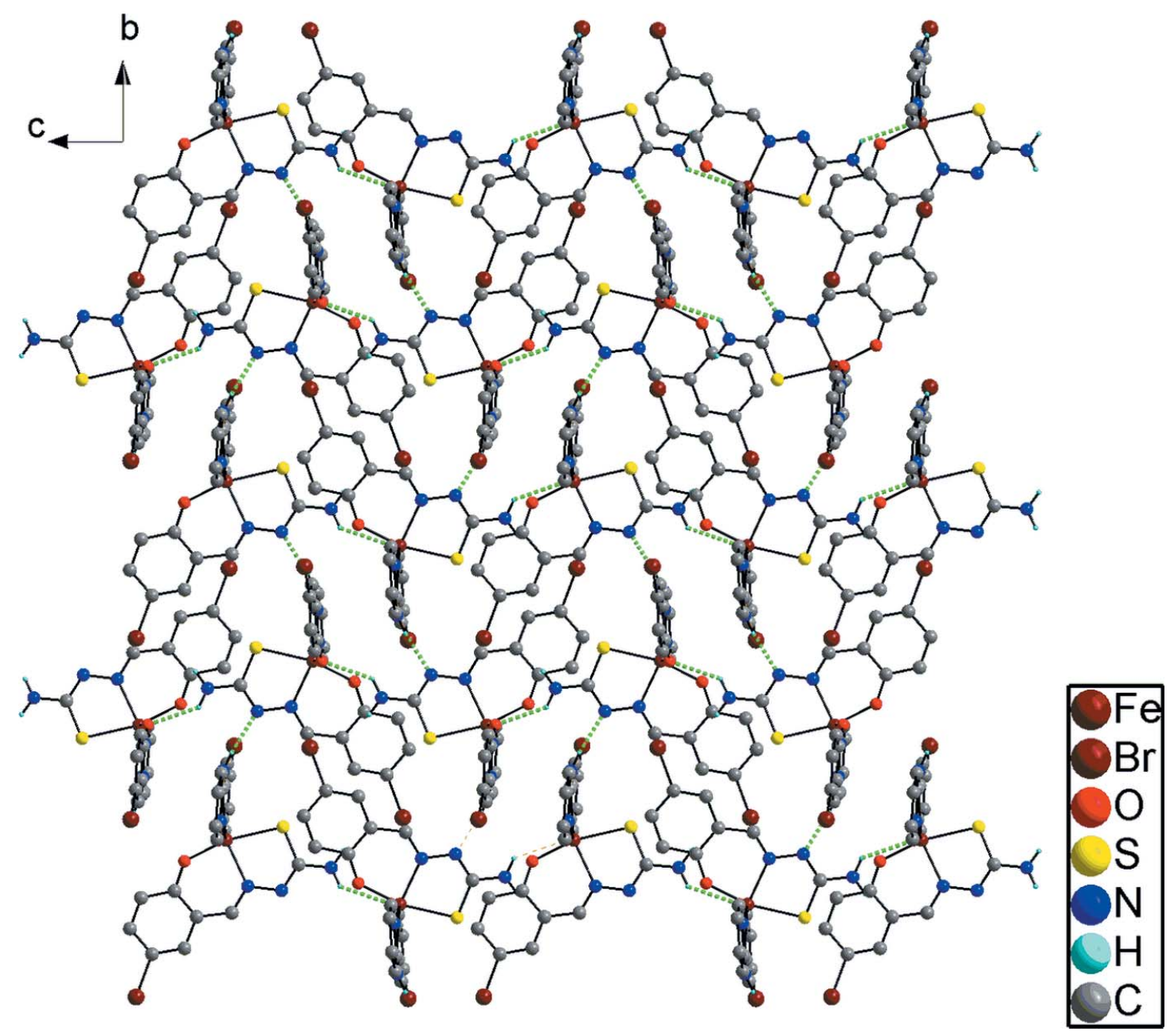

Figure 2

The layered structure of (I) formed through hydrogen bonds (green dotted lines) and $\pi-\pi$ interactions.

\section{Synthesis and crystallization}

All reactions were conducted in air using reagent grade solvents. The 5-Br-Hpsal and 5- $\mathrm{Br}-\mathrm{H}_{2}$ thsa ligands were

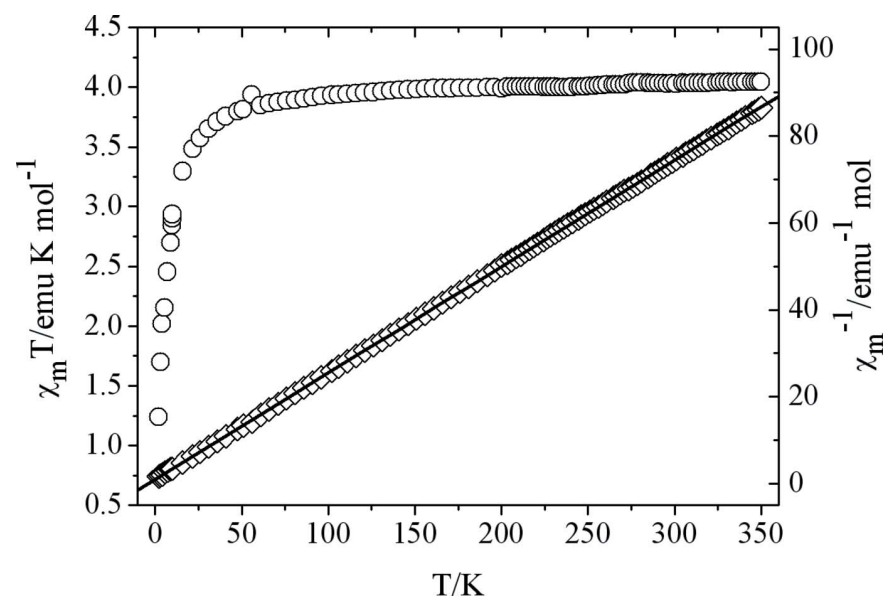

Figure 3

Temperature dependencies of $\chi_{\mathrm{m}} T$ and $\chi_{\mathrm{m}}$ versus temperature (T) for complex (I) measured under an applied field of 2000 Oe. The solid line represents the fitting curve based on the Curie-Weiss law. synthesized by refluxing equimolar 5-bromosalicylaldehyde with thiosemicarbazone and 2-pyridylhydrazine, respectively, in an ethanol solvent. All other chemicals were purchased from the Sigma Aldrich Chemical Company and used as received. The precursors $\left[\mathrm{Fe}(5-\mathrm{Br}-\mathrm{psal})_{2}\right] \mathrm{Cl}$ and $\mathrm{Li}[\mathrm{Fe}(5-\mathrm{Br}-$ thsa $)_{2}$ ] were prepared according to literature methods (Phonsri et al., 2016). $\left[\mathrm{Fe}(5-\mathrm{Br}-\mathrm{psal})_{2}\right] \mathrm{Cl}(0.2 \mathrm{mmol})$ and $\mathrm{Li}\left[\mathrm{Fe}(5-\mathrm{Br} \text {-thsa })_{2}\right](0.2 \mathrm{mmol})$ were dissolved in acetonitrile $(20 \mathrm{~mL})$. The mixture was filtered and kept at room temperature for two days. Brown block-shaped single crystals were collected with a relatively high yield of $76 \%$. Elemental analysis calculated for $\mathrm{C}_{20} \mathrm{H}_{15} \mathrm{~N}_{6} \mathrm{O}_{2} \mathrm{SBr}_{2} \mathrm{Fe}: \mathrm{C}, 38.80 \% ; \mathrm{H}$, $2.44 \%$; N, $13.57 \%$; found: C, 38.72\%, H, 2.38\%; N, $13.62 \%$.

\section{Refinement}

Crystal data, data collection and structure refinement details are summarized in Table 2. The amino- $\mathrm{H}$ atom of 5-Br-psal ${ }^{-}$ was found from the difference-Fourier map and refined isotropically. All other hydrogen atoms were placed in calculated positions with $\mathrm{C}-\mathrm{H}=0.88-0.95 \AA$ and refined in the 
Table 2

Experimental details.

\begin{tabular}{|c|c|}
\hline \multicolumn{2}{|l|}{ Crystal data } \\
\hline Chemical formula & {$\left[\mathrm{Fe}\left(\mathrm{C}_{8} \mathrm{H}_{6} \mathrm{BrN}_{3} \mathrm{OS}\right)\left(\mathrm{C}_{12} \mathrm{H}_{9} \mathrm{BrN}_{3} \mathrm{O}\right)\right]$} \\
\hline$M_{\mathrm{r}}$ & 619.11 \\
\hline Crystal system, space group & Monoclinic, $C 2 / c$ \\
\hline Temperature $(\mathrm{K})$ & 123 \\
\hline$a, b, c(\AA)$ & $21.145(4), 14.738(3), 15.471(3)$ \\
\hline$\beta\left({ }^{\circ}\right)$ & $112.47(3)$ \\
\hline$V\left(\AA^{3}\right)$ & $4455.2(18)$ \\
\hline$Z$ & 8 \\
\hline Radiation type & Мо $K \alpha$ \\
\hline$\mu\left(\mathrm{mm}^{-1}\right)$ & 4.39 \\
\hline Crystal size $(\mathrm{mm})$ & $0.12 \times 0.10 \times 0.08$ \\
\hline \multicolumn{2}{|l|}{ Data collection } \\
\hline Diffractometer & $\begin{array}{l}\text { Bruker APEXII CCD area- } \\
\text { detector }\end{array}$ \\
\hline Absorption correction & $\begin{array}{l}\text { Multi-scan (CrystalClear; Rigaku, } \\
\text { 2008) }\end{array}$ \\
\hline$T_{\min }, T_{\max }$ & $0.576,0.707$ \\
\hline $\begin{array}{l}\text { No. of measured, independent and } \\
\text { observed }[I>2 \sigma(I)] \text { reflections }\end{array}$ & $17937,5012,3514$ \\
\hline$R_{\text {int }}$ & 0.074 \\
\hline$(\sin \theta / \lambda)_{\max }\left(\AA^{-1}\right)$ & 0.649 \\
\hline \multicolumn{2}{|l|}{ Refinement } \\
\hline$R\left[F^{2}>2 \sigma\left(F^{2}\right)\right], w R\left(F^{2}\right), S$ & $0.048,0.106,1.00$ \\
\hline No. of reflections & 5012 \\
\hline No. of parameters & 293 \\
\hline $\mathrm{H}$-atom treatment & $\begin{array}{l}\mathrm{H} \text { atoms treated by a mixture of } \\
\text { independent and constrained } \\
\text { refinement }\end{array}$ \\
\hline$\Delta \rho_{\max }, \Delta \rho_{\min }\left(\mathrm{e} \AA^{-3}\right)$ & $0.53,-0.76$ \\
\hline
\end{tabular}

Computer programs: CrystalClear (Rigaku, 2008), SHELXS97 and SHELXTL (Sheldrick, 2008), SHELXL2014 (Sheldrick, 2015) and publCIF (Westrip, 2010). riding model with fixed isotropic displacement parameters $\left[U_{\text {iso }}(\mathrm{H})=1.2 U_{\text {eq }}(\mathrm{C}, \mathrm{N})\right]$.

\section{Funding information}

The work was supported by the Fundamental Research Funds for the Central Universities (No. 2015QNA24).

\section{References}

Li, Z. Y., Dai, J. W., Shiota, Y., Yoshizawa, K., Kanegawa, S. \& Sato, O. (2013). Chem. Eur. J. 19, 12948-12952.

Li, G.-L. \& Sato, O. (2017). Acta Cryst. E73, 993-995.

Ni, Z.-H., Xu, L., Li, N. \& Zhang, L. F. (2017). Inorg. Chim. Acta, 462 , 204-208.

Phonsri, W., Davies, C. G., Jameson, G. N. L., Moubaraki, B. \& Murray, K. S. (2016). Chem. Eur. J. 22, 1322-1333.

Phonsri, W., Harding, P., Murray, K. S., Moubaraki, B. \& Harding, D. J. (2017). New J. Chem. 41, 13747-13753.

Rigaku (2008). CrystalClear. Rigaku Corporation, Tokyo, Japan.

Sato, O., Tao, J. \& Zhang, Y.-Z. (2007). Angew. Chem. Int. Ed. 46, 2152-2187.

Sheldrick, G. M. (2008). Acta Cryst. A64, 112-122.

Sheldrick, G. M. (2015). Acta Cryst. C71, 3-8.

Shongwe, M. S., Al-Barhi, K. S., Mikuriya, M., Adams, H., Morris, M. J., Bill, E. \& Molloy, K. C. (2014). Chem. Eur. J. 20, 9693-9701. Westrip, S. P. (2010). J. Appl. Cryst. 43, 920-925.

Zhang, R., Xu, L., Ni, Z., Chen, H. \& Zhang, L. (2016). Inorg. Chem. Commun. 67, 99-102. 


\section{supporting information}

Acta Cryst. (2018). E74, 252-255 [https://doi.org/10.1107/S2056989018001263]

\section{A new mononuclear neutral high-spin iron(III) complex with the different} tridentate ligands 5-bromosalicylaldehyde (pyridin-2-yl)hydrazone and 5bromosalicylaldehyde thiosemicarbazone

\section{Yun Zhao, Xiao-Feng Shen and Li-Fang Zhang}

Computing details

Data collection: CrystalClear (Rigaku, 2008); cell refinement: CrystalClear (Rigaku, 2008); data reduction: CrystalClear (Rigaku, 2008); program(s) used to solve structure: SHELXS97 (Sheldrick, 2008); program(s) used to refine structure: SHELXL2014 (Sheldrick, 2015); molecular graphics: SHELXTL (Sheldrick, 2008); software used to prepare material for publication: publCIF (Westrip, 2010).

[1-(5-Bromo-2-oxidobenzylidene)thiosemicarbazidato](4-bromo-2-\{[2-(pyridin-2-

yl)hydrazinylidene]methyl\}phenolato)iron(III)

Crystal data

$\left[\mathrm{Fe}\left(\mathrm{C}_{8} \mathrm{H}_{6} \mathrm{BrN}_{3} \mathrm{OS}\right)\left(\mathrm{C}_{12} \mathrm{H}_{9} \mathrm{BrN}_{3} \mathrm{O}\right)\right]$

$M_{r}=619.11$

Monoclinic, $C 2 / c$

$a=21.145(4) \AA$

$b=14.738(3) \AA$

$c=15.471(3) \AA$

$\beta=112.47(3)^{\circ}$

$V=4455.2(18) \AA^{3}$

$Z=8$

\section{Data collection}

Bruker APEXII CCD area-detector diffractometer

Radiation source: Rotating Anode $\omega$ scans

Absorption correction: multi-scan

(CrystalClear; Rigaku, 2008)

$T_{\min }=0.576, T_{\max }=0.707$

17937 measured reflections

\section{Refinement}

Refinement on $F^{2}$

Least-squares matrix: full

$R\left[F^{2}>2 \sigma\left(F^{2}\right)\right]=0.048$

$w R\left(F^{2}\right)=0.106$

$S=1.00$

5012 reflections

293 parameters
$F(000)=2440$

$D_{\mathrm{x}}=1.846 \mathrm{Mg} \mathrm{m}^{-3}$

Mo $K \alpha$ radiation, $\lambda=0.71073 \AA$

Cell parameters from 2456 reflections

$\theta=3.0-26.6^{\circ}$

$\mu=4.39 \mathrm{~mm}^{-1}$

$T=123 \mathrm{~K}$

Block, brown

$0.12 \times 0.10 \times 0.08 \mathrm{~mm}$

5012 independent reflections

3514 reflections with $I>2 \sigma(I)$

$R_{\text {int }}=0.074$

$\theta_{\max }=27.5^{\circ}, \theta_{\min }=3.1^{\circ}$

$h=-27 \rightarrow 25$

$k=-18 \rightarrow 19$

$l=-20 \rightarrow 18$

0 restraints

Primary atom site location: difference Fourier map

Secondary atom site location: difference Fourier map

Hydrogen site location: mixed 
$\mathrm{H}$ atoms treated by a mixture of independent and constrained refinement

$w=1 /\left[\sigma^{2}\left(F_{\mathrm{o}}^{2}\right)+(0.0455 P)^{2}+0.2543 P\right]$

where $P=\left(F_{\mathrm{o}}^{2}+2 F_{\mathrm{c}}^{2}\right) / 3$

$$
\begin{aligned}
& (\Delta / \sigma)_{\max }=0.001 \\
& \Delta \rho_{\max }=0.53 \mathrm{e} \AA^{-3} \\
& \Delta \rho_{\min }=-0.76 \mathrm{e} \AA^{-3}
\end{aligned}
$$

\section{Special details}

Geometry. All esds (except the esd in the dihedral angle between two 1.s. planes) are estimated using the full covariance matrix. The cell esds are taken into account individually in the estimation of esds in distances, angles and torsion angles; correlations between esds in cell parameters are only used when they are defined by crystal symmetry. An approximate

\begin{tabular}{|c|c|c|c|c|}
\hline & $x$ & $y$ & $z$ & $U_{\text {iso }} * / U_{\text {eq }}$ \\
\hline $\mathrm{Fe} 1$ & $0.25435(3)$ & 0.91027 (4) & $0.12510(4)$ & $0.01599(15)$ \\
\hline $\mathrm{Br} 1$ & $0.11616(2)$ & $1.34761(3)$ & -0.13350 & $0.02748(14)$ \\
\hline $\mathrm{Br} 2$ & $0.56554(3)$ & $0.64748(4)$ & $0.14892(4)$ & $0.04080(16)$ \\
\hline O1 & $0.20626(15)$ & 0.96909 (19) & $0.00536(18)$ & $0.0228(7)$ \\
\hline $\mathrm{O} 2$ & $0.33707(15)$ & $0.91116(19)$ & $0.1001(2)$ & $0.0226(7)$ \\
\hline $\mathrm{S} 1$ & $0.30696(6)$ & $0.87380(7)$ & $0.28908(7)$ & 0.0232 \\
\hline N1 & $0.32499(18)$ & $0.9869(2)$ & $0.4275(2)$ & $0.0250(8)$ \\
\hline H1A & 0.3225 & 1.0394 & 0.4532 & $0.030^{*}$ \\
\hline H1B & 0.3446 & 0.9402 & 0.4631 & $0.030 *$ \\
\hline N2 & $0.27026(18)$ & $1.0507(2)$ & $0.2850(2)$ & $0.0184(8)$ \\
\hline N3 & $0.24650(17)$ & $1.0386(2)$ & $0.1873(2)$ & $0.0160(7)$ \\
\hline N4 & $0.26090(17)$ & $0.7660(2)$ & $0.1140(2)$ & $0.0165(7)$ \\
\hline N5 & $0.20634(19)$ & $0.7168(2)$ & $0.1166(2)$ & $0.0212(8)$ \\
\hline N6 & $0.15575(18)$ & $0.8575(2)$ & $0.1092(2)$ & $0.0198(8)$ \\
\hline $\mathrm{C} 1$ & 0.2987 (2) & $0.9781(3)$ & $0.3335(3)$ & $0.0183(9)$ \\
\hline $\mathrm{C} 2$ & $0.2241(2)$ & $1.1126(3)$ & $0.1416(3)$ & $0.0191(9)$ \\
\hline $\mathrm{H} 2$ & 0.2272 & 1.1656 & 0.1780 & $0.023 *$ \\
\hline $\mathrm{C} 3$ & 0.1945 (2) & $1.1242(3)$ & $0.0406(3)$ & $0.0171(9)$ \\
\hline $\mathrm{C} 4$ & $0.1734(2)$ & $1.2118(3)$ & $0.0059(3)$ & $0.0190(9)$ \\
\hline $\mathrm{H} 4$ & 0.1792 & 1.2605 & 0.0484 & $0.023^{*}$ \\
\hline $\mathrm{C} 5$ & $0.1448(2)$ & $1.2278(3)$ & $-0.0876(3)$ & $0.0182(9)$ \\
\hline C6 & $0.1360(2)$ & $1.1570(3)$ & $-0.1512(3)$ & $0.0252(10)$ \\
\hline H6 & 0.1161 & 1.1686 & -0.2165 & $0.030 *$ \\
\hline $\mathrm{C} 7$ & $0.1558(2)$ & $1.0713(3)$ & $-0.1194(3)$ & $0.0246(10)$ \\
\hline $\mathrm{H} 7$ & 0.1490 & 1.0235 & -0.1632 & $0.030 *$ \\
\hline $\mathrm{C} 8$ & $0.1863(2)$ & $1.0516(3)$ & $-0.0228(3)$ & $0.0170(9)$ \\
\hline C9 & $0.3869(2)$ & 0.8521 & $0.1141(3)$ & $0.0211(10)$ \\
\hline $\mathrm{C} 10$ & $0.4514(2)$ & $0.8828(3)$ & $0.1204(3)$ & $0.0272(10)$ \\
\hline H10 & 0.4588 & 0.9460 & 0.1170 & $0.033^{*}$ \\
\hline C11 & $0.5042(2)$ & $0.8236(3)$ & 0.1311 (3) & $0.0298(11)$ \\
\hline H11 & 0.5477 & 0.8453 & 0.1361 & $0.036^{*}$ \\
\hline $\mathrm{C} 12$ & $0.4922(2)$ & $0.7305(3)$ & $0.1347(3)$ & $0.0295(11)$ \\
\hline $\mathrm{C} 13$ & $0.4306(2)$ & $0.6972(3)$ & $0.1282(3)$ & $0.0241(10)$ \\
\hline H13 & 0.4240 & 0.6335 & 0.1299 & $0.029 *$ \\
\hline $\mathrm{C} 14$ & $0.3763(2)$ & $0.7575(3)$ & $0.1191(3)$ & $0.0207(9)$ \\
\hline
\end{tabular}
(isotropic) treatment of cell esds is used for estimating esds involving l.s. planes.

Fractional atomic coordinates and isotropic or equivalent isotropic displacement parameters $\left(\AA^{2}\right)$ 


\begin{tabular}{lllll} 
C15 & $0.3135(2)$ & $0.7189(3)$ & $0.1168(3)$ & $0.0202(9)$ \\
H15 & 0.3101 & 0.6546 & 0.1172 & $0.024^{*}$ \\
C16 & $0.1507(2)$ & $0.7671(3)$ & $0.1137(3)$ & $0.0209(9)$ \\
C17 & $0.0910(2)$ & $0.7237(3)$ & $0.1113(3)$ & $0.0279(11)$ \\
H17 & 0.0889 & 0.6595 & 0.1157 & $0.034^{*}$ \\
C18 & $0.0352(2)$ & $0.7776(3)$ & $0.1022(3)$ & $0.0315(11)$ \\
H18 & -0.0060 & 0.7505 & 0.1005 & $0.038^{*}$ \\
C19 & $0.0396(2)$ & $0.8719(3)$ & $0.0956(3)$ & $0.0312(11)$ \\
H19 & 0.0016 & 0.9099 & 0.0885 & $0.037^{*}$ \\
C20 & $0.1007(2)$ & $0.9079(3)$ & $0.0996(3)$ & $0.0269(10)$ \\
H20 & 0.1041 & 0.9718 & 0.0953 & $0.032^{*}$ \\
H5 & $0.214(2)$ & $0.665(3)$ & $0.140(3)$ & $0.022(13)^{*}$ \\
\hline
\end{tabular}

Atomic displacement parameters $\left(\AA^{2}\right)$

\begin{tabular}{|c|c|c|c|c|c|c|}
\hline & $U^{11}$ & $U^{22}$ & $U^{33}$ & $U^{12}$ & $U^{13}$ & $U^{23}$ \\
\hline $\mathrm{Fe} 1$ & $0.0230(3)$ & $0.0106(3)$ & 0.0143 (3) & $0.0020(3)$ & $0.0071(3)$ & $-0.0004(2)$ \\
\hline $\mathrm{Br} 1$ & 0.0358 (3) & $0.0187(2)$ & $0.0243(2)$ & $0.0050(2)$ & $0.0074(2)$ & $0.00645(19)$ \\
\hline $\mathrm{Br} 2$ & 0.0304 (3) & $0.0502(4)$ & 0.0369 (3) & $0.0172(2)$ & $0.0073(2)$ & $-0.0101(2)$ \\
\hline O1 & $0.0372(19)$ & $0.0142(15)$ & $0.0146(15)$ & $0.0049(13)$ & $0.0073(14)$ & $0.0016(12)$ \\
\hline $\mathrm{O} 2$ & $0.0283(17)$ & $0.0176(15)$ & $0.0253(16)$ & $0.0048(14)$ & $0.0140(14)$ & $0.0042(13)$ \\
\hline S1 & $0.0350(7)$ & $0.0156(5)$ & $0.0160(5)$ & $0.0058(5)$ & $0.0063(5)$ & -0.0004 \\
\hline N1 & 0.037 (2) & 0.019 (2) & $0.0144(18)$ & $0.0031(17)$ & $0.0049(17)$ & $0.0010(15)$ \\
\hline N2 & $0.029(2)$ & $0.0158(18)$ & $0.0109(16)$ & $0.0016(15)$ & $0.0085(15)$ & $-0.0026(14)$ \\
\hline N3 & 0.0220 (19) & $0.0145(18)$ & $0.0129(17)$ & $0.0002(15)$ & $0.0082(15)$ & $-0.0029(13)$ \\
\hline N4 & 0.0217 (19) & $0.0160(18)$ & $0.0098(16)$ & $-0.0018(15)$ & $0.0038(14)$ & $-0.0010(13)$ \\
\hline N5 & $0.032(2)$ & $0.0092(18)$ & 0.0214 (19) & $-0.0001(16)$ & $0.0096(17)$ & $0.0003(15)$ \\
\hline N6 & 0.0219 (19) & $0.019(2)$ & $0.0152(18)$ & $-0.0012(16)$ & $0.0035(15)$ & $-0.0012(14)$ \\
\hline $\mathrm{C} 1$ & $0.024(2)$ & $0.014(2)$ & $0.018(2)$ & $-0.0017(18)$ & 0.0093 (19) & $-0.0021(16)$ \\
\hline $\mathrm{C} 2$ & $0.026(2)$ & $0.013(2)$ & $0.020(2)$ & $0.0003(18)$ & $0.0116(19)$ & $-0.0010(17)$ \\
\hline $\mathrm{C} 3$ & $0.022(2)$ & $0.016(2)$ & $0.014(2)$ & $0.0054(18)$ & $0.0071(18)$ & $0.0024(16)$ \\
\hline $\mathrm{C} 4$ & $0.033(3)$ & $0.011(2)$ & $0.014(2)$ & $0.0027(18)$ & $0.0101(19)$ & $0.0021(16)$ \\
\hline $\mathrm{C} 5$ & $0.020(2)$ & $0.014(2)$ & $0.021(2)$ & $0.0034(17)$ & $0.0072(18)$ & $0.0052(17)$ \\
\hline C6 & $0.030(3)$ & $0.029(3)$ & $0.015(2)$ & $0.004(2)$ & 0.0070 (19) & $0.0036(19)$ \\
\hline $\mathrm{C} 7$ & $0.034(3)$ & $0.026(3)$ & $0.012(2)$ & $0.004(2)$ & 0.0065 (19) & $-0.0018(18)$ \\
\hline $\mathrm{C} 8$ & 0.018 (2) & $0.015(2)$ & 0.018 (2) & $0.0013(17)$ & $0.0056(18)$ & $0.0027(16)$ \\
\hline $\mathrm{C} 9$ & $0.029(3)$ & $0.021(2)$ & $0.014(2)$ & $0.009(2)$ & 0.0096 (19) & $0.0027(17)$ \\
\hline $\mathrm{C} 10$ & $0.031(3)$ & $0.029(3)$ & $0.023(2)$ & $-0.001(2)$ & $0.011(2)$ & $-0.001(2)$ \\
\hline C11 & $0.023(3)$ & 0.040 & $0.025(2)$ & $0.000(2)$ & 0.007 (2) & $-0.003(2)$ \\
\hline $\mathrm{C} 12$ & $0.029(3)$ & $0.035(3)$ & $0.024(2)$ & $0.012(2)$ & $0.009(2)$ & $-0.004(2)$ \\
\hline $\mathrm{C} 13$ & $0.032(3)$ & $0.018(2)$ & $0.022(2)$ & $0.004(2)$ & $0.010(2)$ & $-0.0044(18)$ \\
\hline C14 & $0.027(2)$ & $0.023(2)$ & $0.011(2)$ & $0.0045(19)$ & $0.0060(18)$ & $-0.0031(17)$ \\
\hline $\mathrm{C} 15$ & $0.029(3)$ & $0.012(2)$ & $0.013(2)$ & $0.0022(18)$ & $0.0000(18)$ & $-0.0017(16)$ \\
\hline $\mathrm{C} 16$ & $0.030(2)$ & $0.021(2)$ & $0.0089(19)$ & $-0.003(2)$ & $0.0035(18)$ & $-0.0010(16)$ \\
\hline $\mathrm{C} 17$ & $0.034(3)$ & $0.028(3)$ & $0.024(2)$ & -0.008 & $0.012(2)$ & $-0.006(2)$ \\
\hline $\mathrm{C} 18$ & $0.027(3)$ & 0.035 & 0.030 & $-0.009(2)$ & $0.008(2)$ & $-0.002(2)$ \\
\hline C19 & $0.026(3)$ & $0.036(3)$ & $0.029(3)$ & $0.004(2)$ & 0.007 (2) & $-0.001(2)$ \\
\hline $\mathrm{C} 20$ & $0.029(3)$ & $0.027(2)$ & $0.023(2)$ & 0.005 & 0.009 (2) & $-0.002(2)$ \\
\hline
\end{tabular}


Geometric parameters $\left(\AA,{ }^{\circ}\right)$

\begin{tabular}{|c|c|c|c|}
\hline $\mathrm{Fe} 1-\mathrm{O} 2$ & $1.931(3)$ & $\mathrm{C} 4-\mathrm{C} 5$ & $1.359(5)$ \\
\hline $\mathrm{Fe} 1-\mathrm{O} 1$ & $1.943(3)$ & $\mathrm{C} 4-\mathrm{H} 4$ & 0.9500 \\
\hline $\mathrm{Fe} 1-\mathrm{N} 4$ & $2.142(3)$ & $\mathrm{C} 5-\mathrm{C} 6$ & $1.396(6)$ \\
\hline $\mathrm{Fe} 1-\mathrm{N} 6$ & $2.150(4)$ & $\mathrm{C} 6-\mathrm{C} 7$ & $1.362(6)$ \\
\hline $\mathrm{Fe} 1-\mathrm{N} 3$ & $2.157(3)$ & $\mathrm{C} 6-\mathrm{H} 6$ & 0.9500 \\
\hline $\mathrm{Fe} 1-\mathrm{S} 1$ & $2.4093(14)$ & $\mathrm{C} 7-\mathrm{C} 8$ & $1.412(5)$ \\
\hline $\mathrm{Br} 1-\mathrm{C} 5$ & $1.913(4)$ & $\mathrm{C} 7-\mathrm{H} 7$ & 0.9500 \\
\hline $\mathrm{Br} 2-\mathrm{C} 12$ & $1.921(4)$ & $\mathrm{C} 9-\mathrm{C} 10$ & $1.405(6)$ \\
\hline $\mathrm{O} 1-\mathrm{C} 8$ & $1.307(5)$ & $\mathrm{C} 9-\mathrm{C} 14$ & $1.418(6)$ \\
\hline $\mathrm{O} 2-\mathrm{C} 9$ & $1.318(5)$ & $\mathrm{C} 10-\mathrm{C} 11$ & $1.376(6)$ \\
\hline $\mathrm{S} 1-\mathrm{C} 1$ & $1.720(4)$ & $\mathrm{C} 10-\mathrm{H} 10$ & 0.9500 \\
\hline $\mathrm{N} 1-\mathrm{C} 1$ & $1.350(5)$ & $\mathrm{C} 11-\mathrm{C} 12$ & $1.399(6)$ \\
\hline $\mathrm{N} 1-\mathrm{H} 1 \mathrm{~A}$ & 0.8800 & $\mathrm{C} 11-\mathrm{H} 11$ & 0.9500 \\
\hline N1-H1B & 0.8800 & $\mathrm{C} 12-\mathrm{C} 13$ & $1.361(6)$ \\
\hline $\mathrm{N} 2-\mathrm{C} 1$ & $1.314(5)$ & $\mathrm{C} 13-\mathrm{C} 14$ & $1.416(6)$ \\
\hline $\mathrm{N} 2-\mathrm{N} 3$ & $1.409(4)$ & $\mathrm{C} 13-\mathrm{H} 13$ & 0.9500 \\
\hline $\mathrm{N} 3-\mathrm{C} 2$ & $1.287(5)$ & $\mathrm{C} 14-\mathrm{C} 15$ & $1.433(6)$ \\
\hline $\mathrm{N} 4-\mathrm{C} 15$ & $1.298(5)$ & $\mathrm{C} 15-\mathrm{H} 15$ & 0.9500 \\
\hline $\mathrm{N} 4-\mathrm{N} 5$ & $1.376(5)$ & $\mathrm{C} 16-\mathrm{C} 17$ & $1.403(6)$ \\
\hline $\mathrm{N} 5-\mathrm{C} 16$ & $1.377(6)$ & $\mathrm{C} 17-\mathrm{C} 18$ & $1.384(6)$ \\
\hline N5-H5 & $0.83(4)$ & C17-H17 & 0.9500 \\
\hline $\mathrm{N} 6-\mathrm{C} 20$ & $1.339(5)$ & $\mathrm{C} 18-\mathrm{C} 19$ & $1.400(6)$ \\
\hline $\mathrm{N} 6-\mathrm{C} 16$ & $1.341(5)$ & $\mathrm{C} 18-\mathrm{H} 18$ & 0.9500 \\
\hline $\mathrm{C} 2-\mathrm{C} 3$ & $1.454(5)$ & $\mathrm{C} 19-\mathrm{C} 20$ & $1.376(6)$ \\
\hline $\mathrm{C} 2-\mathrm{H} 2$ & 0.9500 & C19-H19 & 0.9500 \\
\hline $\mathrm{C} 3-\mathrm{C} 4$ & $1.403(5)$ & $\mathrm{C} 20-\mathrm{H} 20$ & 0.9500 \\
\hline $\mathrm{C} 3-\mathrm{C} 8$ & $1.416(5)$ & & \\
\hline $\mathrm{O} 2-\mathrm{Fe} 1-\mathrm{O} 1$ & $89.41(12)$ & $\mathrm{C} 4-\mathrm{C} 5-\mathrm{Br} 1$ & $120.3(3)$ \\
\hline $\mathrm{O} 2-\mathrm{Fe} 1-\mathrm{N} 4$ & $84.23(12)$ & $\mathrm{C} 6-\mathrm{C} 5-\mathrm{Br} 1$ & $119.4(3)$ \\
\hline $\mathrm{O} 1-\mathrm{Fe} 1-\mathrm{N} 4$ & $113.13(12)$ & $\mathrm{C} 7-\mathrm{C} 6-\mathrm{C} 5$ & $119.9(4)$ \\
\hline $\mathrm{O} 2-\mathrm{Fe} 1-\mathrm{N} 6$ & $153.25(13)$ & $\mathrm{C} 7-\mathrm{C} 6-\mathrm{H} 6$ & 120.0 \\
\hline $\mathrm{O} 1-\mathrm{Fe} 1-\mathrm{N} 6$ & $85.48(13)$ & $\mathrm{C} 5-\mathrm{C} 6-\mathrm{H} 6$ & 120.0 \\
\hline $\mathrm{N} 4-\mathrm{Fe} 1-\mathrm{N} 6$ & $73.81(13)$ & $\mathrm{C} 6-\mathrm{C} 7-\mathrm{C} 8$ & $121.7(4)$ \\
\hline $\mathrm{O} 2-\mathrm{Fe} 1-\mathrm{N} 3$ & $108.28(13)$ & $\mathrm{C} 6-\mathrm{C} 7-\mathrm{H} 7$ & 119.2 \\
\hline $\mathrm{O} 1-\mathrm{Fe} 1-\mathrm{N} 3$ & $86.13(12)$ & $\mathrm{C} 8-\mathrm{C} 7-\mathrm{H} 7$ & 119.2 \\
\hline $\mathrm{N} 4-\mathrm{Fe} 1-\mathrm{N} 3$ & $157.59(12)$ & $\mathrm{O} 1-\mathrm{C} 8-\mathrm{C} 7$ & $120.1(4)$ \\
\hline $\mathrm{N} 6-\mathrm{Fe} 1-\mathrm{N} 3$ & 97.57 (13) & $\mathrm{O} 1-\mathrm{C} 8-\mathrm{C} 3$ & $122.3(4)$ \\
\hline $\mathrm{O} 2-\mathrm{Fe} 1-\mathrm{S} 1$ & $97.11(10)$ & $\mathrm{C} 7-\mathrm{C} 8-\mathrm{C} 3$ & $117.6(4)$ \\
\hline $\mathrm{O} 1-\mathrm{Fe} 1-\mathrm{S} 1$ & $165.00(9)$ & $\mathrm{O} 2-\mathrm{C} 9-\mathrm{C} 10$ & $119.5(4)$ \\
\hline $\mathrm{N} 4-\mathrm{Fe} 1-\mathrm{S} 1$ & $81.07(9)$ & $\mathrm{O} 2-\mathrm{C} 9-\mathrm{C} 14$ & $121.7(4)$ \\
\hline $\mathrm{N} 6-\mathrm{Fe} 1-\mathrm{S} 1$ & $94.42(10)$ & $\mathrm{C} 10-\mathrm{C} 9-\mathrm{C} 14$ & $118.7(4)$ \\
\hline $\mathrm{N} 3-\mathrm{Fe} 1-\mathrm{S} 1$ & $79.01(9)$ & $\mathrm{C} 11-\mathrm{C} 10-\mathrm{C} 9$ & $121.7(4)$ \\
\hline $\mathrm{C} 8-\mathrm{O} 1-\mathrm{Fe} 1$ & $135.9(3)$ & $\mathrm{C} 11-\mathrm{C} 10-\mathrm{H} 10$ & 119.1 \\
\hline $\mathrm{C} 9-\mathrm{O} 2-\mathrm{Fe} 1$ & $133.6(3)$ & $\mathrm{C} 9-\mathrm{C} 10-\mathrm{H} 10$ & 119.1 \\
\hline $\mathrm{C} 1-\mathrm{S} 1-\mathrm{Fe} 1$ & $98.35(14)$ & $\mathrm{C} 10-\mathrm{C} 11-\mathrm{C} 12$ & $118.5(4)$ \\
\hline
\end{tabular}




$$
\begin{aligned}
& \mathrm{C} 1-\mathrm{N} 1-\mathrm{H} 1 \mathrm{~A} \\
& \mathrm{C} 1-\mathrm{N} 1-\mathrm{H} 1 \mathrm{~B} \\
& \mathrm{H} 1 \mathrm{~A}-\mathrm{N} 1-\mathrm{H} 1 \mathrm{~B} \\
& \mathrm{C} 1-\mathrm{N} 2-\mathrm{N} 3 \\
& \mathrm{C} 2-\mathrm{N} 3-\mathrm{N} 2 \\
& \mathrm{C} 2-\mathrm{N} 3-\mathrm{Fe} 1 \\
& \mathrm{~N} 2-\mathrm{N} 3-\mathrm{Fe} 1 \\
& \mathrm{C} 15-\mathrm{N} 4-\mathrm{N} 5 \\
& \mathrm{C} 15-\mathrm{N} 4-\mathrm{Fe} 1 \\
& \mathrm{~N} 5-\mathrm{N} 4-\mathrm{Fe} 1 \\
& \mathrm{~N} 4-\mathrm{N} 5-\mathrm{C} 16 \\
& \mathrm{~N} 4-\mathrm{N} 5-\mathrm{H} 5 \\
& \mathrm{C} 16-\mathrm{N} 5-\mathrm{H} 5 \\
& \mathrm{C} 20-\mathrm{N} 6-\mathrm{C} 16 \\
& \mathrm{C} 20-\mathrm{N} 6-\mathrm{Fe} 1 \\
& \mathrm{C} 16-\mathrm{N} 6-\mathrm{Fe} 1 \\
& \mathrm{~N} 2-\mathrm{C} 1-\mathrm{N} 1 \\
& \mathrm{~N} 2-\mathrm{C} 1-\mathrm{S} 1 \\
& \text { N1-C1-S1 } \\
& \text { N3-C2-C3 } \\
& \text { N3-C2-H2 } \\
& \text { C3-C2-H2 } \\
& \text { C4-C3-C8 } \\
& \text { C4-C3-C2 } \\
& \text { C8-C3-C2 } \\
& \text { C5-C4-C3 } \\
& \text { C5-C4-H4 } \\
& \text { C3- } 4-\mathrm{H} 4 \\
& \text { C4-C5-C6 }
\end{aligned}
$$

120.0

120.0

120.0

$114.1(3)$

$112.8(3)$

$125.0(3)$

$122.2(2)$

$115.8(4)$

$127.5(3)$

$116.1(2)$

$115.6(4)$

$118(3)$

$122(3)$

$118.2(4)$

$125.1(3)$

$116.6(3)$

$116.6(4)$

$126.4(3)$

$117.0(3)$

$127.3(4)$

116.4

116.4

$119.5(4)$

$117.5(4)$

$123.0(4)$

$121.0(4)$

119.5

119.5

$120.3(4)$
$\mathrm{C} 10-\mathrm{C} 11-\mathrm{H} 11$

C12-C11-H11

$\mathrm{C} 13-\mathrm{C} 12-\mathrm{C} 11$

$\mathrm{C} 13-\mathrm{C} 12-\mathrm{Br} 2$

$\mathrm{C} 11-\mathrm{C} 12-\mathrm{Br} 2$

$\mathrm{C} 12-\mathrm{C} 13-\mathrm{C} 14$

$\mathrm{C} 12-\mathrm{C} 13-\mathrm{H} 13$

C14-C13-H13

C13-C14-C9

$\mathrm{C} 13-\mathrm{C} 14-\mathrm{C} 15$

C9-C14-C15

$\mathrm{N} 4-\mathrm{C} 15-\mathrm{C} 14$

N4-C15-H15

C14-C15-H15

N6- C16-N5

N6-C16- 17

$\mathrm{N} 5-\mathrm{C} 16-\mathrm{C} 17$

$\mathrm{C} 18-\mathrm{C} 17-\mathrm{C} 16$

C18-C17-H17

C16-C17-H17

C17-C18-C19

C17-C18-H18

C19-C18-H18

$\mathrm{C} 20-\mathrm{C} 19-\mathrm{C} 18$

$\mathrm{C} 20-\mathrm{C} 19-\mathrm{H} 19$

$\mathrm{C} 18-\mathrm{C} 19-\mathrm{H} 19$

$\mathrm{N} 6-\mathrm{C} 20-\mathrm{C} 19$

$\mathrm{N} 6-\mathrm{C} 20-\mathrm{H} 20$

$\mathrm{C} 19-\mathrm{C} 20-\mathrm{H} 20$
120.8

120.8

$122.2(4)$

119.1 (4)

$118.6(4)$

$119.8(4)$

120.1

120.1

119.0 (4)

$117.4(4)$

$123.5(4)$

$124.2(4)$

117.9

117.9

$116.9(4)$

$122.8(4)$

120.3 (4)

$117.6(4)$

121.2

121.2

120.0 (4)

120.0

120.0

$117.8(4)$

121.1

121.1

$123.6(5)$

118.2

118.2

Hydrogen-bond geometry $\left(\AA,{ }^{\circ}\right)$

\begin{tabular}{lllll}
\hline$D-\mathrm{H}^{\cdots} \cdots A$ & $D-\mathrm{H}$ & $\mathrm{H} \cdots A$ & $D \cdots A$ & $D-\mathrm{H} \cdots A$ \\
\hline $\mathrm{N} 5-\mathrm{H} 5 \cdots \mathrm{N} 2^{\mathrm{i}}$ & $0.83(4)$ & $2.00(4)$ & $2.825(5)$ & $171(4)$ \\
$\mathrm{N} 1-\mathrm{H} 1 A \cdots \mathrm{O} 2^{\mathrm{ii}}$ & 0.88 & 2.29 & $2.987(4)$ & 136 \\
\hline
\end{tabular}

Symmetry codes: (i) $-x+1 / 2, y-1 / 2,-z+1 / 2$; (ii) $x,-y+2, z+1 / 2$. 